\title{
Not Available
}

National Cancer Institute

\section{Source}

National Cancer Institute. Not Available. NCI Thesaurus. Code C126101.

The desired information is not available. 